\title{
具有侧挂胆固醇液晶元的两亲嵌段功能大分子的合成及自组装研究
}

\author{
胡方振 $a, b \quad$ 陈圣典 ${ }^{b}$ 李慧 $b \quad$ 孙景景 ${ }^{b}$ 盛瑞隆 ${ }^{b}$ \\ 罗挺 $b$ 曹阿民*,b \\ ( ${ }^{a}$ 华东理工大学 材料科学与工程学院 上海 200237) \\ ${ }^{b}$ 中国科学院上海有机化学研究所 高分子材料研究室 上海 200032)
}

\begin{abstract}
摘要 采用可逆加成-断裂链转移(RAFT)聚合法, 合成了系列具有刚性疏水胆固醇液晶元的聚甲基丙烯酸甘油酯-嵌段聚甲基丙烯酸亚已基胆固醇酯(PGMA- $b$-PMA6Chol)两亲嵌段功能大分子. 运用核磁共振(NMR)和凝胶渗透色谱(GPC) 表征了其化学结构及分子量, 并对其热性质、液晶相结构及相转变行为分别运用热台偏振光显微镜(POM)、热重分析 仪(TGA)、示差扫描量热仪(DSC)和二维小角 X 射线散射(2D-SAXS) 表征. 采用纳米沉淀法研究了所得嵌段大分子的溶 液自组装, 动态光散射(DLS)和扫描电子显微镜(SEM)的研究发现溶液自组装聚集体为尺寸 $0.7 \sim 2.0 \mu \mathrm{m}$ 的球形结构, 其中含有较高刚性链段质量比例的嵌段大分子组装形成开口中空结构的聚集体，且其尺寸随着溶液温度的升高减小， 呈现可逆温度变化响应性. 结果表明刚性疏水胆固醇液晶单元和具有多羟基结构的亲水性甲基丙烯酸甘油酯的嵌段共 聚可以调控该类嵌段大分子自组装及溶液聚集体形貌.
\end{abstract}

关键词＼cjkstart可逆加成-断裂链转移聚合; 两亲性; 胆固醇液晶元；自组装

\section{Preparation of New Amphiphilic Liquid-Crystal Diblock Copolymers Bearing Side-on Cholesteryl Mesogen and Their Self-aggregation}

\author{
Hu, Fangzhen ${ }^{a, b}$ \\ Chen, Shengdian ${ }^{b}$ \\ Luo, Ting $^{b}$ \\ Li, Hui ${ }^{b} \quad$ Sun, Jingjing ${ }^{b}$ \\ Sheng, Ruilong ${ }^{b}$ \\ ( ${ }^{a}$ School of Materials Science and Engineering, East China University of Science and Technology, Shanghai 200237) \\ ( ${ }^{b}$ Laboratory for Polymer Materials, Shanghai Institute of Organic Chemistry, Chinese Academy of Sciences, \\ Shanghai 200032)
}

\begin{abstract}
Amphiphilic block copolymers bearing rigid hydrophobic liquid crystal mesogen have recently attracted broad interests since they could spontaneously self-assemble into functional objects at the micron and/or nanometer scales, and these soft matters bearing high-order hierarchical structures have potential applications in micro-reactors, advanced catalysts, biosensors, drug delivery, biotechnology and so forth. In this work, we designed and successfully prepared a new series of amphiphilic liquid crystal poly(glyceryl methacrylate)- $b$-poly(6-cholesteryloxyhexyl methacrylate) (PGMA- $b$-PMA6Chol) with hydrophobic PMA6Chol block bearing end-on cholesteryl mesogen through sequential controlled reversible additionfragmentation chain transfer polymerization (RAFT) and successive removal of ketal protection groups. Then, their structures and comonomer composition were examined by nuclear magnetic resonance (NMR) and gel permeation chromatography (GPC). Liquid crystal phase structures and their transition as well as thermal stability of the prepared LC copolymers were characterized by differential scanning calorimeter (DSC), polarized optical microscope (POM), 2D small angle X-ray scattering (2D-SAXS) and thermogravimetric analytic instrument (TGA). Moreover, self-aggregation of these amphiphilic LC block copolymers in mixed solution was investigated by means of UV spectrometer, and the aggregate sizes and morphologies were examined by scanning electron microscopy (SEM) and dynamic light scattering (DLS), respectively. As a result, it was revealed that the LC to isotropic phase transition temperature $\left(T_{\mathrm{LC}-\mathrm{I}}\right)$ of as-synthesized LC block copolymers increased with an increase in rigid LC block content, and the presence of bishydroxyl groups of hydrophilic PGMA strongly influenced the self-assembly and LC phase of rigid hydrophobic block in bulk state. In mixed solvent, the synthesized amphiphilic LC copolymers were found to preferably self-aggregate into core-shell spherical objects with average sizes spanning a range from 0.7 to $2.0 \mu \mathrm{m}$ when the hydrophobic LC block content was less than $93 \%$, in contrast, the LC copolymer with $93 \mathrm{wt} \%$ LC block tended to form open hollow spherical aggregates, showing interesting thermal responsive particle sizes in aqueous solution.
\end{abstract}

Keywords controlled polymerization; LC block copolymer; amphiphilic polymer; cholesteryl mesogen; self-assembly

*E-mail: acao@mail.sioc.ac.cn; Tel.: 021-54925303

Received December 27, 2012; published January 23, 2013.

Project supported by the National Natural Science Foundation of China (Nos. 20874114, 21174160, 21002116).

项目受国家自然科学基金(Nos. 20874114, 21174160, 21002116)资助. 


\section{1 引言}

两亲性嵌段功能大分子因其结构中同时具有亲水/ 疏水链段以及嵌段单元间可能发生相分离的特性, 使得 其在凝聚态或选择性溶剂中能够通过非共价键相互作 用, 自组织形成多尺度的有序结构聚集体, 并由此作为 一种具有多尺度和多层次结构功能新材料的高效构建 手段, 在当今功能材料化学、高效药物输送载体、生物 医学工程等领域得到广泛关注与应用 ${ }^{[1 \sim 3]}$. 具有空间有 序取向能力和刚性链段的液晶大分子嵌段共聚物, 通过 自组装能够形成有序的凝聚态物理结构和微/纳米结构 形貌. 液晶功能大分子及其液晶元的链段流动性, 可以 对外部的热、光、电、磁等信号产生响应性, 促使其作 为两亲性嵌段功能大分子的重要构筑砌块, 进一步应用 于分子设计和构建新型有机功能软物质 ${ }^{[4]}$.

到目前为止已有的液晶嵌段大分子的相关文献报 道中, 偶氮苯、芳香多环结构为报道较多的液晶元 ${ }^{[5]}$. 研 究发现由上述液晶元制备的液晶功能大分子对热、光、 电、磁等外源刺激信号具有灵敏的响应性 ${ }^{[6,7]}$. 另一方面, 面向生物医学工程应用, 基于上述分子结构的液晶嵌段 功能大分子普遍存在细胞毒性高、生物相容性差等不足, 制约了其在药物输送、生物医学工程、膜结构仿生等领 域的应用. 与之相比较, 最早发现的液晶功能分子之一 一天然产物胆固醇具有多环刚性分子骨架, 是构成细 胞生物膜和脂质不可缺少的成分 ${ }^{[8]}$. 最近的研究发现其 在细胞内外的胆固醇代谢及胆固醇浓度水平调控过程 中发挥重要的作用 ${ }^{[9]}$, 并可应用于化学合成高效、低毒 的新型基因输送功能脂质体 ${ }^{[10]}$. 因此, 设计采用具有胆 固醇液晶元的疏水单元, 通过可控共聚合, 制备新型两 亲性液晶嵌段功能大分子, 将为发展具有外部刺激响应 性的生物功能材料提供新的可能.

围绕含有胆固醇液晶元的两亲嵌段液晶功能大分 子的制备与自聚集行为研究, 本组与居里研究所李敏慧 小组前期的合作研究中, 发现以系列分子量甲基封端聚 乙二醇(MPEG)为亲水嵌段构建的两亲胆固醇液晶嵌段 功能大分子在选择性溶剂中自组装形成具有多级结构 的纳米纤维、囊泡、中空纳米管等, 刚性胆固醇液晶元 有序堆砌与预组装诱导嵌段功能大分子的本体和溶液 自组织聚集行为 ${ }^{[11,12]}$. 为了进一步拓展该系列胆固醇液 晶嵌段大分子的生物材料应用, 本组最近报道了具有自 由羟基的聚甲基丙烯酸羟乙酯(PHEMA)为亲水性嵌段 两亲胆固醇液晶嵌段功能大分子, 有趣地发现液晶嵌段 的相转变温度 $\left(T_{\mathrm{LC}-\mathrm{I}}\right)$ 高于胆固醇液晶均聚物, 揭示亲水 嵌段氢键网络的存在, 可能在嵌段共聚物微相分离的过 程中诱导液晶的有序取向与排列 ${ }^{[13]}$. 另一方面, 重复单 元侧基含有双羟基的聚甲基丙烯酸甘油酯(PGMA)具有 更优异的亲水性和生物相容性, 大量的羟基可共价偶联 药物或生物活性分子，同时已经在隐形眼镜、水凝胶、 药物输送载体等领域得到关注与应用 ${ }^{[14,15]}$, 并进一步拓
展其作为生物相容性人工肾滤过膜材料的应用 ${ }^{[16]}$.

在本组已有相关工作的基础上，为了进一步研究阐 明亲水嵌段中多羟基官能团的存在、氢键网络密度对胆 固醇液晶嵌段功能大分子聚集行为、液晶相结构与转变 及其溶液自组装的影响规律, 本文新采用生物基甘油为 合成出发原料，以缩酮保护的甲基丙烯酸丙酮缩甘油酯 (SMA)和含有胆固醇液晶元的甲基丙烯酸亚已基胆固 醇酯(MA6Chol)作为共聚单体, 通过顺序可逆加成-断 裂链转移聚合(RAFT) 反应和去保护反应 ${ }^{[17,18]}$, 合成制 备了系列结构规整、窄分子量分布的两亲性聚甲基丙烯 酸甘油酯-嵌段-聚甲基丙烯酸亚已基胆固醇酯 (PGMA- $b$-PMA6Chol), 并进一步对其液晶相及其转 变、凝聚态微结构和溶液自组装聚集行为进行了研究.

\section{2 结果与讨论}

\section{1 单体的合成}

本文采用胆固醇、甲基丙烯酸和己二醇为主要起始 原料，多步合成了以六亚甲基柔性碳氢链为胆固醇液晶 元连接片段的甲基丙烯酸亚已基胆固醇酯(MA6Chol)功 能单体. 经过柱分离、纯化, 所得 $\mathrm{MA} 6 \mathrm{Chol}$ 单体的 ${ }^{1} \mathrm{H}$ NMR 图谱与文献报道一致 ${ }^{[19]}$. 同时, 采用丙酮缩甘油 为原料，合成制备并纯化得到双差基缩酮保护的甲基丙 烯酸缩酮甘油酯(SMA)单体.

\section{2 系列液晶嵌段共聚物的制备}

以二硫代苯甲酸 4-氰基戊酸(CPADB)为链转移剂、 AIBN 为引发剂, 在甲苯溶液中首先进行单体 SMA 的 RAFT 聚合. 反应结束后, 分离、纯化得到粉红色聚合物 PSMA. 相关产物的 ${ }^{1} \mathrm{H}$ NMR 谱图(图 1A)中, $\delta 5.61$ 和 6.16 处 SMA 单体的双键氢核磁特征信号消失, 同时在 $\delta$ 1.90 处出现 PSMA 产物结构骨架亚甲基氢 $\left(\mathrm{CCH}_{2} \mathrm{C}\right)$ 的

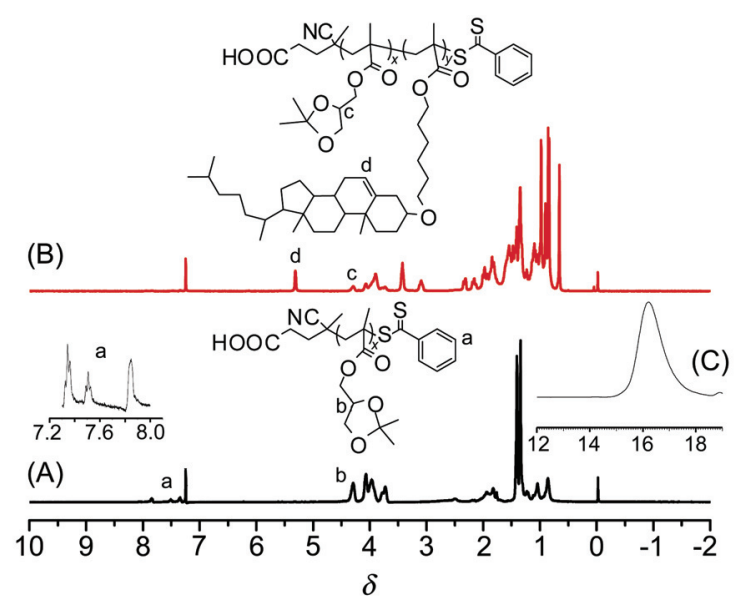

图 1 PSMA (A)和 PSMA $11-b-\mathrm{PMA}_{1} \mathrm{Chol}_{19}$ (B) 的 ${ }^{1} \mathrm{H} \mathrm{NMR}$ 谱图 $\left(\mathrm{CDCl}_{3}\right)$ 以及 PSMA 的 GPC 图(C)

Figure $1{ }^{1} \mathrm{H}$ NMR spectra of PSMA (A) and PSMA $11-b$-PMA6Chol ${ }_{19}$ (B) in $\mathrm{CDCl}_{3}$ as well as GPC curves of the PSMA (C) 
核磁共振信号宽峰. 此外, $\delta 7.35,7.51$ 和 7.84 处出现苯 环氢的核磁共振特征信号 (图 1A-a). 以上结果表明 SMA 单体在 RAFT 试剂的存在下发生自由基聚合. PSMA 产物的 GPC 表征结果如图 $1 \mathrm{C}$ 和表 1 所示, 得到 分子量分布 PDI 为 1.19 , 并且 GPC 信号呈对称形态分布 的单峰, 表明 SMA 单体的聚合为活性 RAFT 聚合. 由聚 合产物 PSMA 的 ${ }^{1} \mathrm{H}$ NMR 谱图(图 1A), 通过 RAFT 试剂 苯环氢的特征核磁共振信号 $\mathrm{a}(\delta 7.3 \sim 7.84)$ 与 $\mathrm{SMA}$ 重复 单元五元环叔碳氢的特征核磁共振信号 $\mathrm{b}(\delta$ 4.30)的积 分强度比, 计算得到 PSMA 的平均聚合度(DP)约为 11 .

然后以所得 PSMA 为大分子起始剂(Macro-RAFT), $\mathrm{AIBN}$ 为引发剂, 甲苯为溶剂, 引发 $\mathrm{MA} 6 \mathrm{Chol}$ 液晶元单<smiles>C=C(C)C(=O)OCC1COC(C)(C)O1</smiles><smiles>N#CC(CCC(=O)O)(SC(=O)O)SC(=S)c1ccccc1</smiles>

CPADB

$$
\text { SMA (1) }
$$
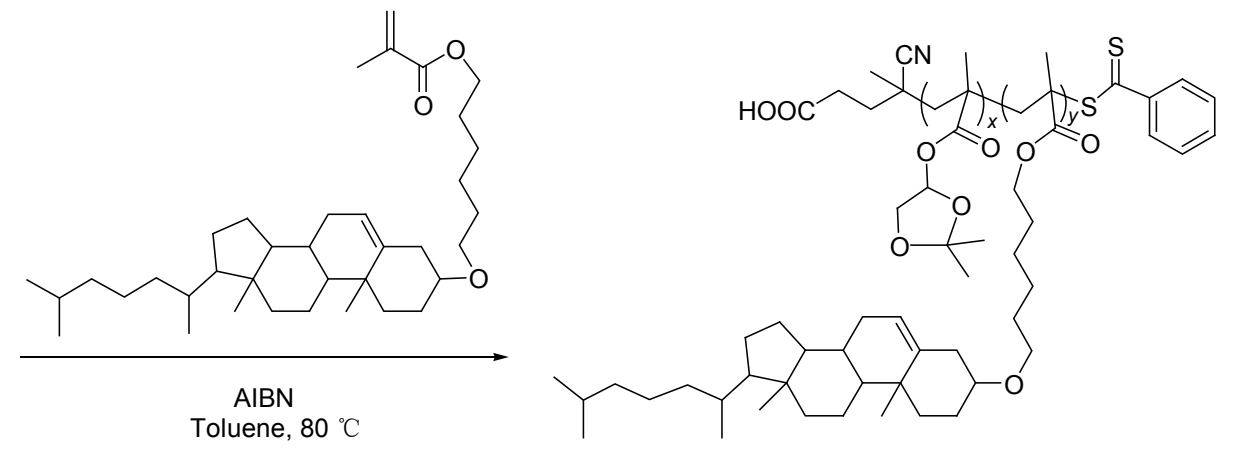

PSMA- $b$-PMA6Chol

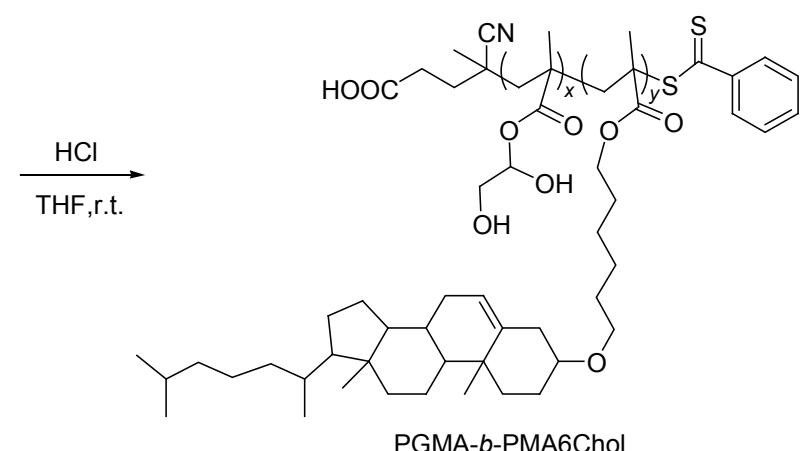

PGMA- $b$-PMA6Chol

图 2 两亲嵌段大分子 PGMA- $b$-PMA6Chol 的合成路线

Figure 2 Synthetic pathway to liquid-crystalline diblock copolymer PGMA- $b$-PMA6Chol

表 1 PSMA, PMA6Chol 及两嵌段大分子 PSMA- $b$-PMA6Chol 的分子量及其分布

Table 1 Molecular weight and distribution of PSMA, PMA6Chol and diblock copolymers

\begin{tabular}{|c|c|c|c|c|c|}
\hline Sample No. & Sample & $\begin{array}{c}M_{\mathrm{n}}(\mathrm{GPC})^{a} / \\
\left(\mathrm{g} \cdot \mathrm{mol}^{-1}\right)\end{array}$ & PDI & $\begin{array}{c}M_{\mathrm{n}}\left({ }^{1} \mathrm{H} \mathrm{NMR}^{b} /\right. \\
\left(\mathrm{g} \cdot \mathrm{mol}^{-1}\right)\end{array}$ & $\begin{array}{c}\text { Hydrophilic/Hydrophobic } \\
\text { weight ratio/wt } \%\end{array}$ \\
\hline- & $\mathrm{PSMA}_{11}$ & 2698 & 1.19 & 2479 & - \\
\hline - & PMA6Chol $_{19}$ & $10510^{c}$ & 1.21 & 一 & 一 \\
\hline $\mathrm{P} 1$ & PSMA $_{11}-b$-PMA6Chol 15 & 10659 & 1.11 & 10806 & $17 / 83$ \\
\hline $\mathrm{P} 2$ & PSMA $_{11}-b$-PMA6Chol 19 & 12040 & 1.10 & 13026 & $14 / 86$ \\
\hline P3 & $\mathrm{PSMA}_{11}-b$-PMA6Chol ${ }_{45}$ & 18018 & 1.13 & 27456 & $7 / 93$ \\
\hline
\end{tabular}

${ }^{a}$ Molecular weights and their distribution were determined by GPC; ${ }^{b}$ DP of PSMA and diblock copolymers were calculated by ${ }^{1} \mathrm{H}$ NMR; ${ }^{c}$ Molecular weights and their distribution were determined by GPC-SLS. 


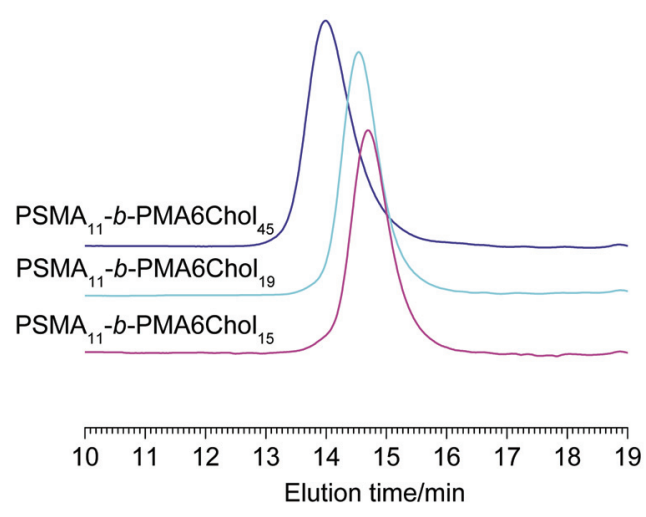

图 3 系列 PSMA- $b$-PMA6Chol 的 GPC 淋洗曲线

Figure 3 GPC curves of the PSMA- $b$-PMA6Chol

PGMA- $b$-PMA6Chol, 其 ${ }^{1} \mathrm{H}$ 核磁共振结构分析结果如图 4 所示. 结果表明在去除保护基后, 由于所得亲水性 PGMA 嵌段在 $\mathrm{CDCl}_{3}$ 中溶解性降低, 形成胆固醇液晶元 嵌段包裹 PGMA 嵌段的反相胶束微结构, 导致只能观 察到 PMA6Chol 液晶嵌段的 ${ }^{1} \mathrm{H}$ NMR 信号(图 4A). 在 $\mathrm{CDCl}_{3} / \mathrm{CD}_{3} \mathrm{OD}$ 的混合溶剂中, PGMA 嵌段呈现一定的溶 解性, 可观察到相应重复单元的 ${ }^{1} \mathrm{H}$ NMR 特征信号, 表 明实现 PSMA 嵌段的羟基完全去保护(图 4B). 在本组之 前基于 MPEG、PHEMA 作为亲水嵌段的液晶嵌段大分 子的工作基础上 ${ }^{[11 ~ 13]}$, 本文以具有双羟基官能团的 PGMA 为亲水嵌段, 进一步研究含有多羟基官能团的亲 水嵌段对液晶大分子聚集和溶液自组装的影响.

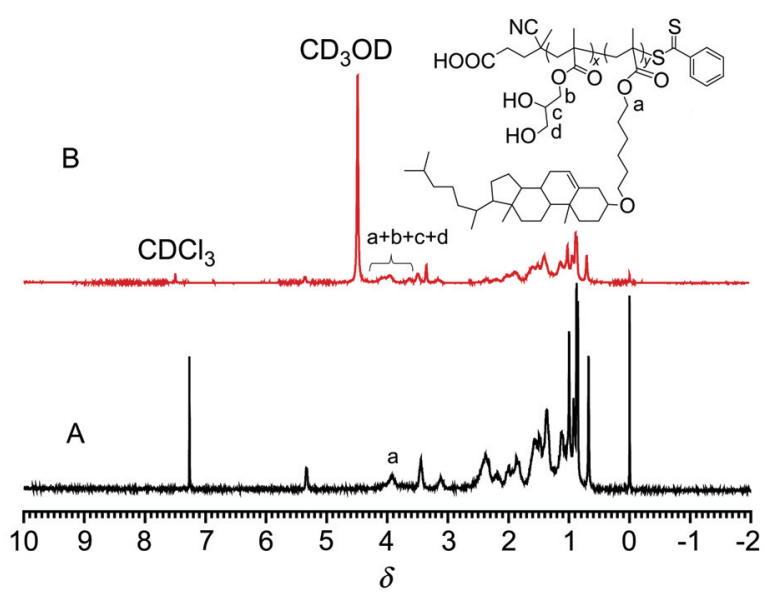

图 $4 \mathrm{PGMA}_{11}-b-\mathrm{PMA} 6 \mathrm{Chol}_{19}$ 在(A) $\mathrm{CDCl}_{3}$ 和(B) $\mathrm{CDCl}_{3} / \mathrm{CD}_{3} \mathrm{OD}$ 中的核 磁谱图

Figure $4{ }^{1} \mathrm{H}$ NMR spectra of $\mathrm{PGMA}_{11}-b$-PMA6Chol ${ }_{19}$ in (A) $\mathrm{CDCl}_{3}$ and (B) $\mathrm{CDCl}_{3} / \mathrm{CD}_{3} \mathrm{OD}$

\section{3 两亲液晶嵌段功能大分子的热物理性质及本体聚 集态结构研究}

在制备所得系列液晶嵌段质量比例的新型两亲功 能大分子的基础上, 本文进一步对其热物理性质、液晶 相结构及其转变行为以及本体状态下聚集态的结构进
行了研究. 图 5 所示为系列两亲性 $\mathrm{PGMA}_{11}-b$ PMA6Chol 液晶嵌段大分子、水溶性 $\mathrm{PGMA}_{11}$ 均聚物和 $\mathrm{PMA} \mathrm{Chol}_{19}$ 液晶均聚物在高纯氮气氛下的热失重分析 (TGA) 图谱. TGA 结果发现在升温至 $300{ }^{\circ} \mathrm{C}$ 前, PMA6Chol 液晶均聚物未观察到明显的热失重, 进一步 升温至 $300 \sim 450{ }^{\circ} \mathrm{C}$, 液晶均聚物迅速分解失重. 而亲 水性 $\mathrm{PGMA}_{11}$ 的 TGA 分析结果与已有文献报道基本一 致, 其特征是升温过程中出现两个明显的热分解失重阶 段 ${ }^{[22]}$. 在系列嵌段比例的液晶共聚物中, 随着液晶嵌 段比例的质量增加, 热分解失重起始温度明显升高, 当疏水性液晶嵌段的质量比例达到 $93 \mathrm{wt} \%$ (液晶嵌段高 分子 $\mathrm{P} 3$ ) 时, 其热分解失重曲线接近于 $\mathrm{PMA} 6 \mathrm{Chol}_{19}$ 液晶 均聚物. 上述结果表明随着 PMA6Chol 液晶链段的质量 比例增加, 所得液晶嵌段大分子的结构趋于更加稳定.

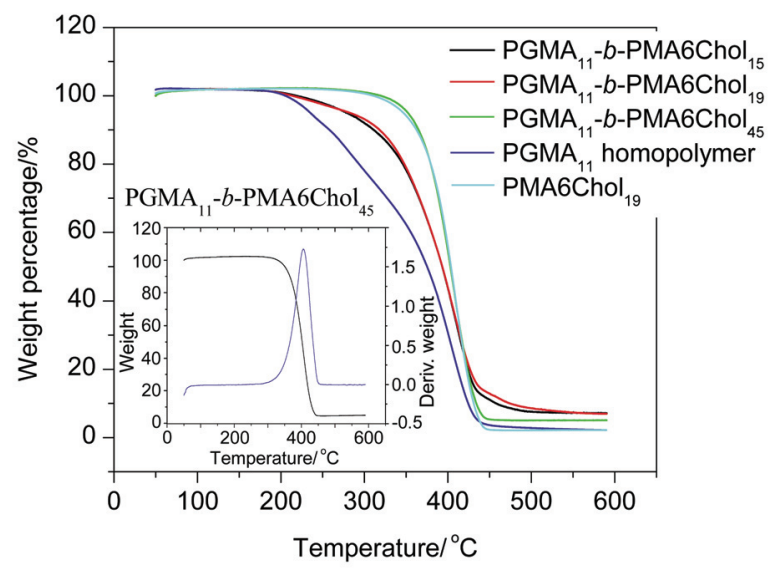

图 5 系列嵌段大分子 $\mathrm{PGMA}_{11}$ - $b$-PMA6Chol, 均聚物 $\mathrm{PMA} 6 \mathrm{Chol}_{19}$ 和 $\mathrm{PGMA}_{11}$ 的 TGA 曲线

Figure 5 TGA traces of the diblock copolymers PGMA $_{11}-b$-PMA6Chol series, homopolymer $\mathrm{PMA} \mathrm{Chol}_{19}$ and $\mathrm{PGMA}_{11}$

双折射是液晶聚合物的一个重要特征光学性质，可 通过热台偏振光显微镜 $(\mathrm{POM})$ 观察液晶聚合物的双折 射、液晶相织构及其转变. 图 6 所示为 $\mathrm{PMA} \mathrm{Chol}{ }_{19}$ 液 晶均聚物及其嵌段共聚物的 $\mathrm{POM}$ 照片, 在 $124{ }^{\circ} \mathrm{C}$ 下 $\mathrm{PMA} \mathrm{Chol}_{19}$ 均聚物可观察到明显的双折射, 并呈近晶 相液晶(Smectic)的扇形织构, 而 $\mathrm{PGMA}_{11}-b-\mathrm{PMA} \mathrm{CChol}_{45}$ (P3)的液晶相织构形成速度较慢, 双折射不明显, 且部 分呈现向列相液晶的织构特征. 结果表明亲水性 $\mathrm{PGMA}_{11}$ 嵌段的存在降低了液晶嵌段大分子中液晶单元 的取向与堆砌有序度.

进一步通过 DSC 研究了系列 PGMA $_{11}-b$-PMA6Chol 的热物理性质以及液晶相转变行为. 首先将样品升温至 $210{ }^{\circ} \mathrm{C}$ 消除热历史, 然后通过降温及升温程序研究其 热物理性质以及液晶相转变行为, 表征结果如图 7 所 示. 结果发现在升温过程中 PMA6Chol 刚性液晶嵌段在 $100 \sim 125{ }^{\circ} \mathrm{C}$ 发生液晶相至各向同性相的转变, 当液晶 嵌段 PMA6Chol 的质量比例由 $86 \mathrm{wt} \%(\mathrm{P} 2)$ 上升至 93 $\mathrm{wt} \%$ (P3)时, PMA6Chol 嵌段的液晶相转变温度 $\left(T_{\mathrm{LC}-\mathrm{I}}\right)$ 由 
$100{ }^{\circ} \mathrm{C}$ 上升至 $125{ }^{\circ} \mathrm{C}$, 而具有较低液晶嵌段质量比例 的 P1 $(83 \mathrm{wt} \%)$ 则观察不到明显的液晶相转变. 上述结 果表明具有较长 PMA6Chol 嵌段的大分子中液晶元趋 于形成有序度更高的堆砌. 同时有趣地发现 PMA6Chol 的 $T_{\mathrm{LC}-\mathrm{I}}$ 为 $130{ }^{\circ} \mathrm{C}$, 明显高于 $\mathrm{PGMA}_{11}-b$-PMA6Chol 的 $T_{\mathrm{LC}-\mathrm{I}}$, 与之前报道的 PMA6Chol- $b$-PHEMA 液晶嵌段大 分子体系呈现完全不同的趋势 ${ }^{[13]}$, 其可能的原因是由 于 PMGA 亲水嵌段中大量羟基官能团的存在增强了亲 水嵌段内的氢键网络相互作用, 制约了 PMA6Chol 链段 的有序排列, 从而导致其 $T_{\mathrm{LC}-\mathrm{I}}$ 低于相应的均聚物, 表明 亲水嵌段的氢键网络密度与作用强度综合作用于疏水 性液晶元的有序排列. 图 8 为 $\mathrm{PGMA}_{11}-b-\mathrm{PMA} \mathrm{C} \mathrm{Chol}_{19}$ (P2)在不同温度下的红外分光谱图, 结果发现随着温度 的升高, 美基的红外吸收峰移向高波数方向, 表明两亲 液晶功能大分子中的氢键作用在高温下被破坏.

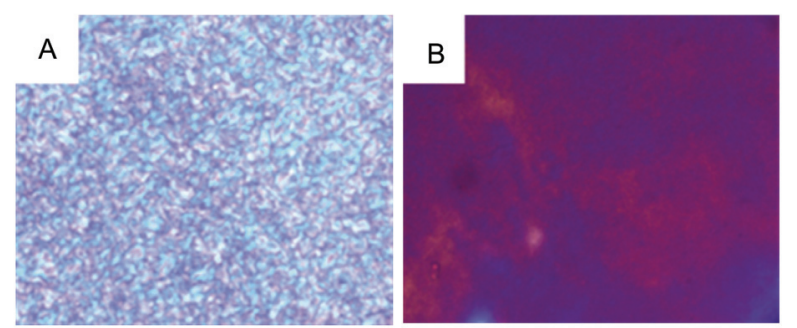

图 6 (A)均聚物 $\mathrm{PMA} \mathrm{Chol}{ }_{19}\left(124{ }^{\circ} \mathrm{C}\right)$ 和(B) $\mathrm{PGMA}_{11}-b$-PMA6Chol 45 (P3) $\left(115{ }^{\circ} \mathrm{C}\right)$ 的 POM 照片

Figure 6 POM photographs of the (A) PMA6Chol ${ }_{19}$ homopolymer (124 ${ }^{\circ} \mathrm{C}$ ) and (B) the $\mathrm{PGMA}_{11}-b$-PMA6Chol ${ }_{45}$ LC diblock copolymers (P3) $\left(115^{\circ} \mathrm{C}\right)$

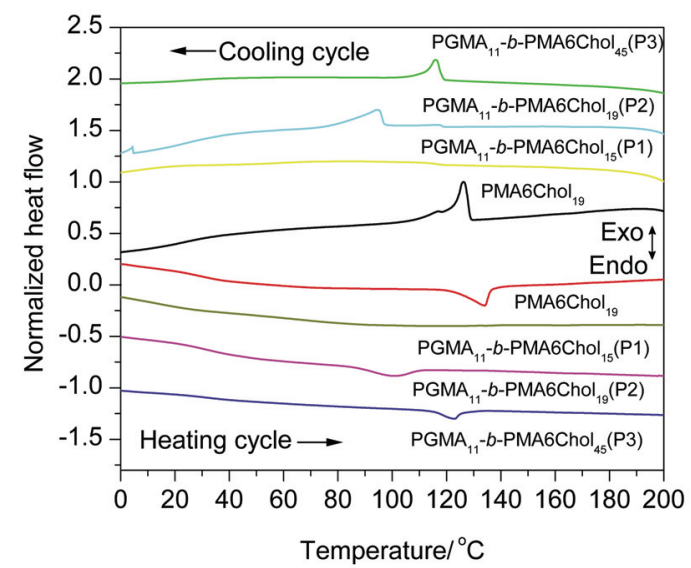

图 7 系列液晶嵌段质量比例的 $\mathrm{PGMA}_{11}-b$-PMA6Chol 和 $\mathrm{PMA} 6 \mathrm{Chol}_{19}$ 均聚物的 DSC 曲线

Figure 7 DSC curves of the diblock copolymers PGMA ${ }_{11}-b$-PMA6Chol series and PMA6Chol ${ }_{19}$ homopolymer

为了进一步阐明 $\mathrm{PGMA}_{11}-b$-PMA6Chol 的液晶相结 构与本体状态下的聚集态结构, 本文进一步采用二维小 角 X射线散射(2D-SAXS)表征所得液晶嵌段功能大分子 聚集态结构, 结果如图 9 所示. 进一步计算得到 $\mathrm{MA} \mathrm{Chol}{ }_{19}$ 均聚物形成近晶型液晶相, 并呈一定的取向
性，规则层状结构周期为 $4.94 \mathrm{~nm}$ ，这一尺寸介于由分 子结构模拟得到的侧链胆甾液晶单元的长度 $l$ 与 $2 l$ 之间 $\left(l\right.$ 约为 $2.7 \mathrm{~nm}^{[19]}$ ), 可确定为 $\mathrm{SmA}_{\mathrm{d}}$ 液晶相 ${ }^{[23]}$, 而共聚物 P3 的 SAXS 散射图案呈弥散状, 取向有序度与均聚物相 比显著下降, 表明多羟基亲水嵌段的引入对胆固醇液晶 元的液晶织构与液晶相转变行为产生重要影响.

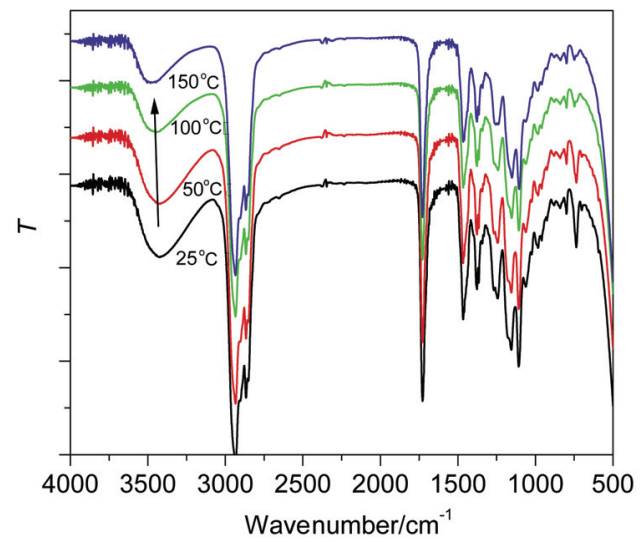

图 8 液晶嵌段大分子 $\mathrm{PGMA}_{11}-b-\mathrm{PMA} \mathrm{Chol}_{19}(\mathrm{P} 2)$ 在不同温度下的红 外光谱图

Figure 8 Temperature-resolved FTIR spectra of the LC diblock copolymer $\mathrm{PGMA}_{11}-b$-PMA6Chol ${ }_{19}(\mathrm{P} 2)$
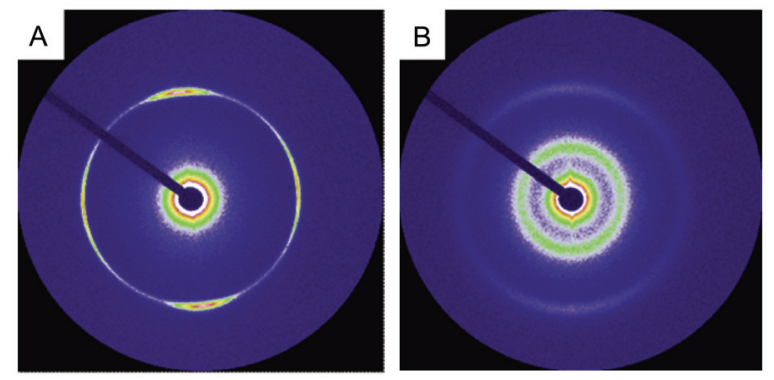

图 9 (A) PMA6Chol 19 均聚物和(B) $\mathrm{PGMA}_{11}-b$-PMA6Chol 45 (P3)嵌段 大分子本体状态下的小角 X-ray 散射图谱

Figure 9 SAXS patterns of the (A) PMA6Chol ${ }_{19}$ homopolymer and (B) the $\mathrm{PGMA}_{11}-b$-PMA6Chol ${ }_{45}(\mathrm{P} 3)$ diblock copolymers in bulk

\section{4 溶液自组装的研究}

为了进一步研究具有刚性液晶嵌段和多羟基亲水嵌 段的两亲液晶功能大分子的自组装, 本文以 THF 作为 两嵌段的共溶剂, 采用 Eisenberg 等 ${ }^{[24,25]}$ 发展的纳米沉 淀法, 研究所得新型液晶功能大分子的自组装及其溶液 聚集体结构. 图 10 为系列 $\mathrm{PGMA}_{11}-b$-PMA6Chol 两亲液 晶嵌段功能大分子溶解于 THF 共溶剂后, 缓慢滴加选 择性溶剂水后所得混合溶液的透光率与水含量的变化 趋势图. 由图 10 中结果可以发现 P1, P2, P3 系列液晶功 能大分子在水含量小于 $7.0 \mathrm{wt} \%$ 时，混合溶液保持澄清 透明，透光率变化幅度小．随着混合溶液含水量的进一 步增加，混合溶液的透光率呈现不同程度的降低，表明 开始形成聚集体，并且随着聚集体形成量的进一步增 加, 混合溶液透光率持续下降. 在实验过程中, 除了 P3 
样品在加水的过程中出现少量的沉淀，导致透光率有所 回升外，其他所有混合溶液体系在含水量大于 $25 \mathrm{wt} \%$ 后, 透光率都趋于稳定, 表明自组装聚集体的形成过程 趋于完成. 进一步加水提升含水量至 $51 \mathrm{wt} \%$, 溶液呈浑 浊状态, 透光率保持稳定. 纳米沉淀自组装过程结束后, 将所得混合溶液在 $25{ }^{\circ} \mathrm{C}$ 条件下在纯水中透析 3 天, 并 通过 DLS 法研究聚集体的平均粒径及其分布, 结果如 图 11 所示. 表明系列 $\mathrm{PGMA}_{11}-b$-PMA6Chol 液晶嵌段共 聚物在 $\mathrm{THF} / \mathrm{water}$ 混合溶剂中自组装形成平均粒径为 $0.7 \sim 2.0 \mu \mathrm{m}$ 的聚集体, 并且 P2 和 P3 样品自组装聚集体 透析液的 DLS 分析结果呈对称单峰. 相比较, P1 样品的 DLS 分析结果为双峰, 导致这一结果的可能原因是 P1 样品的 PMA6Chol 平均链段较短, 且可能存在少量液晶 链段长度低于 $\mathrm{PMA} 6 \mathrm{Chol}$ 平均聚合度, 液晶嵌段大分子 水溶性较强, 趋向于形成粒径更小的胶束聚集体.

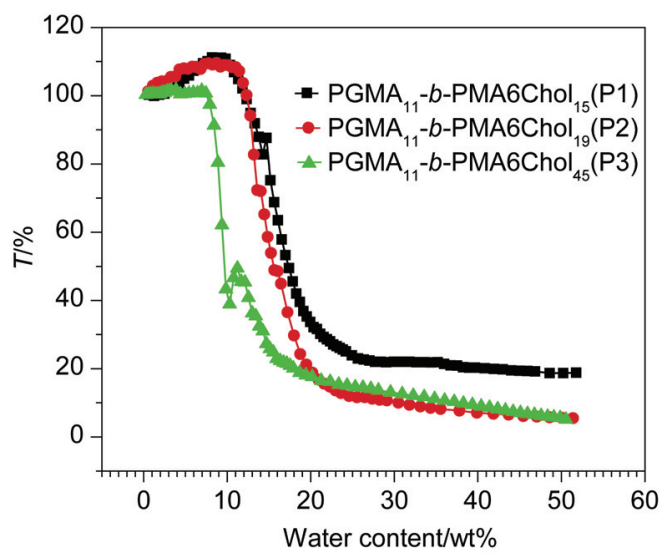

图 10 系列 $\mathrm{PGMA}_{11}-b$-PMA6Chol 液晶嵌段大分子在 THF/water 混合 溶剂中的透光率与水含量的变化关系图

Figure 10 Light transmission as a function of water content in $\mathrm{THF} /$ water mixed solution with $0.1 \mathrm{wt} \%$ initial $\mathrm{PGMA}_{11}-b$-PMA6Chol in THF solution

图 12 为系列 $\mathrm{PGMA}_{11}-b$-PMA6Chol 液晶嵌段大分子 自组装聚集体的 SEM 图像, 聚集体的尺寸与 DLS 法所 测粒径基本一致, 结果表明当两亲嵌段大分子中液晶嵌 段的质量比例较大时(如 P3), 主要形成开口中空形状的 球形聚集体, 并且随着液晶嵌段的质量比例的降低, 自 组装趋于形成实心球聚集体. 到目前为止, 相关体系自 组装形成微米尺度开口中空球形聚集体的报道尚较 少 ${ }^{[26]}$. 关于其可能的形成机理: 由于 PMA6Chol 嵌段具 有较高的玻璃化转变温度 $\left(T_{\mathrm{g}}\right)$, 当向嵌段大分子的 THF 溶液中缓慢加入选择性溶剂水时, 已经溶解的疏水性 PMA6Chol 链段将发生迅速冻结, 形成球状初级聚集体, 进一步加入水后, THF 由疏水内核不断向外抽提, 液晶 嵌段运动完全冻结, 产生聚集体内空腔, 并在空腔薄处 断裂形成最终开口中空球形聚集体. 而具有较短刚性 PMA6Chol 的嵌段大分子由于 PGMA 链段的亲水性作 用, 其液晶链段的冻结不充分, 在加水过程中仍可以发
生链段的移动与调整，导致最终形成实心球形聚集体.
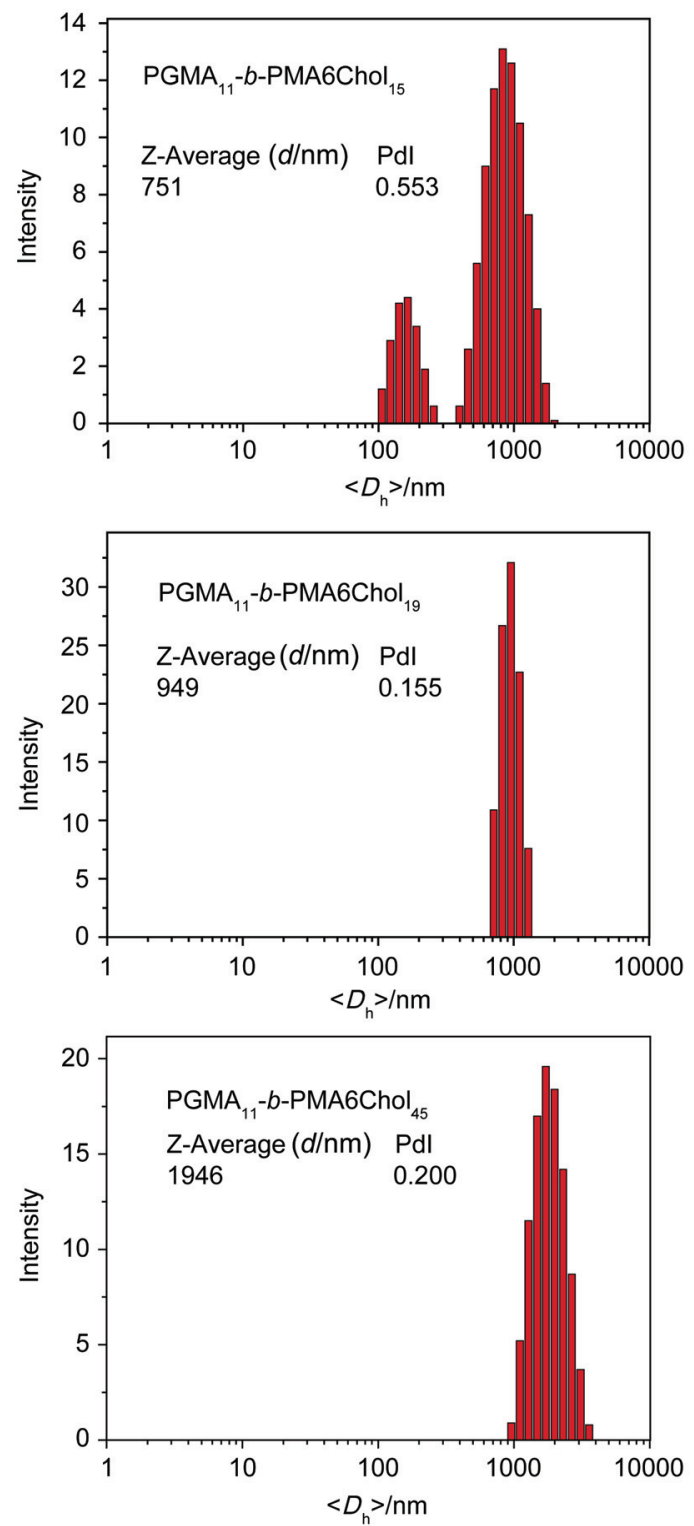

图 11 系列 $\mathrm{PGMA}_{11}-b$-PMA6Chol 样品在 THF/water 中的自组装聚集 体粒径

Figure 11 Hydrodynamic diameter $\left\langle D_{\mathrm{h}}\right\rangle$ of the aggregates selfassembled by the diblock PGMA $11-b$-PMA6Chol in THF/water mixed solvent

基于PGMA亲水性嵌段中含有大量多羟基官能团, 可以相互间或者与水分子间形成氢键相互作用，并容易 受环境温度的影响 ${ }^{[27]}$, 同时随着环境温度的变化, PGMA嵌段的水溶性将发生变化. 图13为两亲液晶嵌段 大分子 $\mathrm{PGMA}_{11}-b-\mathrm{PMA} 6 \mathrm{Chol}_{45}(\mathrm{P} 3,93 \mathrm{wt} \%)$ 纳米沉淀法 自组装所形成的开口中空聚集体在水溶液中平均粒径 随溶液温度变化的趋势图, 结果发现随着温度的升高, 聚集体平均粒径迅速减小, 并具有尺寸变化可逆性. 进 一步如图14所示，当 $60{ }^{\circ} \mathrm{C}$ 条件下的聚集体溶液保持同 样的温度下放置于 $60{ }^{\circ} \mathrm{C}$ 的烘箱干燥后, 聚集体的SEM 
形状如图14A所示, 聚集体呈实心球状, 尺寸显著减小, 与 DLS 的分析结果一致. 当降温至 $25{ }^{\circ} \mathrm{C}$ 时, SEM图像 (图14B)表明聚集体重新恢复为尺寸增大的开口中空形 结构. 关于上述聚集体尺寸温度相应性可逆变化现象, 其可能的解释为: 当温度升高时 PGMA链段的水溶性及 与水分子的氢键作用下降, 导致具有较高弹性的疏水性 液晶球壁发生反方向收缩. 这一有趣现象有望进一步应 用开发作为温敏性的功能载体.
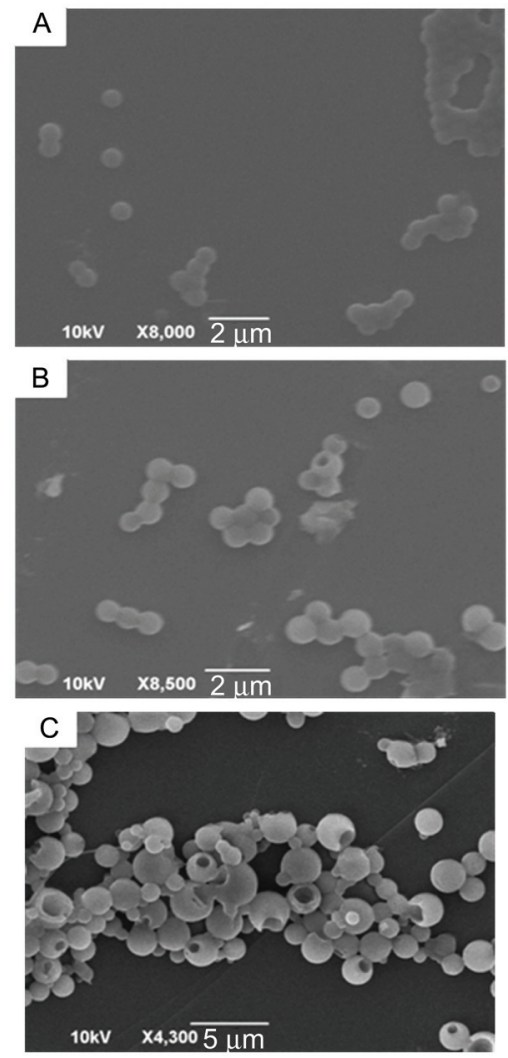

图 12 (A) $\mathrm{PGMA}_{11}-b-\mathrm{PMA} \mathrm{Chol}_{15}$ (P1), (B) $\mathrm{PGMA}_{11}-b$-PMA6Chol 19 (P2)和(C) $\mathrm{PGMA}_{11}-b$-PMA6Chol ${ }_{45}(\mathrm{P} 3)$ 在 $\mathrm{THF} /$ water 混合溶剂中自组装 形成的聚集体 SEM 图像

Figure 12 SEM images of the aggregates formed in THF/water mixed solvent for the diblock (A) $\mathrm{PGMA}_{11}-b-\mathrm{PMA} \mathrm{Chol}_{15}$ (P1), (B) $\mathrm{PGMA}_{11}-b$-PMA6Chol 19 (P2) and (C) $\mathrm{PGMA}_{11}-b$-PMA6Chol 45 (P3)

\section{3 结论}

本文成功合成了一系列结构可控的新型两亲液晶 嵌段聚合物 $\mathrm{PGMA}_{11}-b$-PMA6Chol(P1, P2, P3). ${ }^{1} \mathrm{H}$ NMR 和 GPC 表征结果证明嵌段聚合物结构和 RAFT 活性共 聚合. TGA, DSC 和 SAXS 研究结果发现随着嵌段共聚 物液晶链段质量比例的增加液晶共聚物的热稳定性显 著提升, 同时液晶元在本体状态下的堆砌有序度及液晶 相转变温度增高, 形成层状结构周期为 $4.94 \mathrm{~nm}$ 的近晶 型液晶相结构, 与 PMA6Chol 均聚物相比, PGMA 嵌段 的存在降低了共聚物中液晶嵌段的堆砌有序性. 采用 $\mathrm{THF}$ 为共溶剂, 水为选择性溶剂的纳米沉淀法自组装研 究结果表明, 系列 $\mathrm{PGMA}_{11}-b$-PMA6Chol 在混合溶剂中

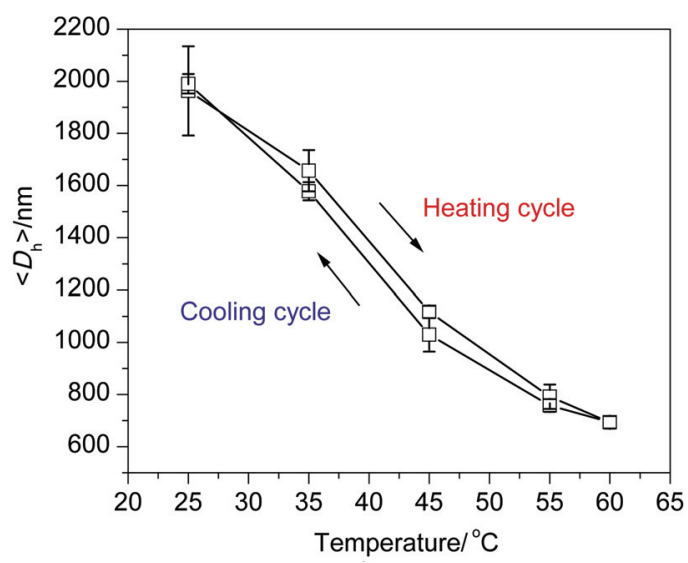

图 $13 \mathrm{PGMA}_{11}-b-\mathrm{PMA} \mathrm{Chol}{ }_{45}(\mathrm{P} 3)$ 自组装聚集体在纯水中粒径随温 度变化的趋势图

Figure 13 Temperature responsive diameter of the $\mathrm{PGMA}_{11}-b$ $\mathrm{PMA6Chol}_{45}$ aggregates in deionized water

表 2 升温-降温循环中 $\mathrm{PGMA}_{11}-b-\mathrm{PMA} \mathrm{C} \mathrm{Chol}_{45}$ (P3)自组装聚集体在 水溶液中的平均粒径及其分布

Table 2 Average diameter of the $\mathrm{PGMA}_{11}-b$-PMA6Chol ${ }_{45}$ (P3) aggregates and their polydispersity indices (PDI) under different temperatures in water

\begin{tabular}{cccccccc}
\hline Heating & $<D_{\mathrm{h}}>/ \mathrm{nm}$ & PDI & & Cooling & $\left\langle D_{\mathrm{h}}>/ \mathrm{nm}\right.$ & PDI \\
\hline $25{ }^{\circ} \mathrm{C}$ & 1963 & 0.096 & & $25{ }^{\circ} \mathrm{C}$ & 1990 & 0.104 \\
$35{ }^{\circ} \mathrm{C}$ & 1657 & 0.085 & & $35{ }^{\circ} \mathrm{C}$ & 1578 & 0.379 \\
$45{ }^{\circ} \mathrm{C}$ & 1117 & 0.280 & & $45{ }^{\circ} \mathrm{C}$ & 1029 & 0.173 \\
$55{ }^{\circ} \mathrm{C}$ & 792 & 0.194 & & $55{ }^{\circ} \mathrm{C}$ & 758 & 0.159 \\
$60{ }^{\circ} \mathrm{C}$ & 693 & 0.189 & & & & \\
\hline
\end{tabular}
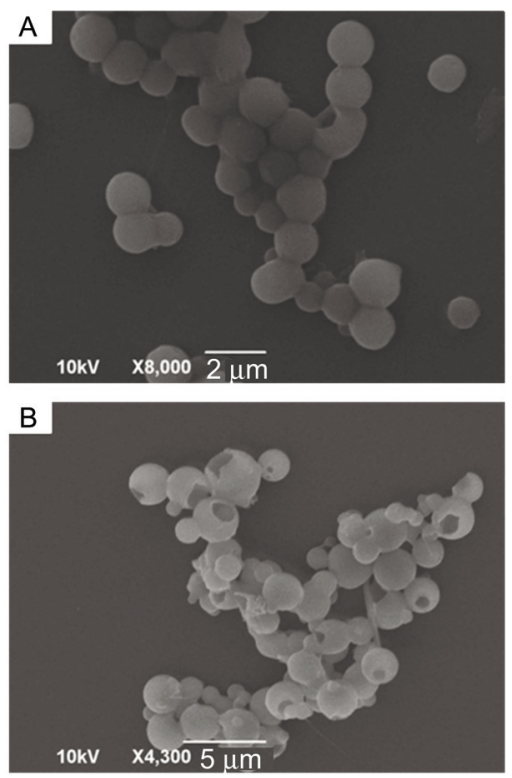

图 $14 \mathrm{PGMA}_{11}-b-\mathrm{PMA} 4 \mathrm{Chol}_{45}$ (P3)自组装聚集体在 $60{ }^{\circ} \mathrm{C}$ (A)和 25 ${ }^{\circ} \mathrm{C}$ (B)下干燥后的 SEM 照片

Figure $14 \mathrm{SEM}$ images of the $\mathrm{PGMA}_{11}-b$-PMA4Chol ${ }_{45}(\mathrm{P} 3)$ aggregates at $60{ }^{\circ} \mathrm{C} \quad(\mathrm{A})$ and $25^{\circ} \mathrm{C}$ (B)

自组装形成了 $0.7 \sim 2.0 \mu \mathrm{m}$ 的球形聚集体, 其中含有 93 $\mathrm{wt} \%$ 疏水性液晶链段的嵌段功能大分子自组装形成开 口中空球形聚集体，并且自组装聚集体在水溶液中随着 
环境温度的升高, 聚集体粒径显著减小, 由开口中空结 构转变为实心球聚集体, 并且尺寸变化具有良好的可逆 性, 有望进一步研究开发成为具有微米尺度的温敏性有 机功能载体.

\section{4 实验部分}

\section{1 试剂}

丙酮缩甘油(aladdin，98\%); 甲基丙烯酰氯(上海达 瑞精细化学有限公司, $98 \%$ ); 偶氮二异丁腈 $(A I B N)($ 上 海试四赫维化工有限公司, CP)经甲醇重结晶三次后使 用; 对甲苯磺酰氯(TCI，98\%)；1，6-己二醇 (TCI，98\%); 二环已基碳二亚胺(DCC)(上海天莲精细化工有限公司, 98\%); 胆固醇(国药集团上海化学试剂有限公司，98\%); 4-二甲氨基吡啶(DMAP)(吉尔生化有限公司, 99\%); 甲 基丙烯酸(上海凌峰化学试剂有限公司, 98\%); 甲苯(国 药集团上海化学试剂有限公司, AR)先经钠丝干燥预处 理, 现蒸现用; 四氢呋喃(金城化工助剂厂, AR).

\section{2 仪器测试与表征}

凝胶渗透色谱仪(GPC, PerkinElmer 200, 美国), 35 ${ }^{\circ} \mathrm{C}$ 下测试, $\mathrm{THF}$ 为流动相, 流速为 $1.0 \mathrm{~mL} / \mathrm{min}$; 核磁共 振仪(NMR, Bruker 300); 热失重分析仪(TGA, TA Q500, 美国), $\mathrm{N}_{2}$ 气氛下 $(60 \mathrm{~mL} / \mathrm{min})$ 升温速率 $20 \mathrm{~K} / \mathrm{min}$; 示差扫 描热分析仪(DSC, TA Q200, 美国), $\mathrm{N}_{2}$ 气氛 $(50 \mathrm{~mL} / \mathrm{min})$ 下进行升温速率为 $10 \mathrm{~K} / \mathrm{min}$ 一次降温扫描和二次升温 扫描测量; 热台偏光显微镜(POM, Olympus BX51), 1 $\mathrm{K} / \mathrm{min}$ 升温熔融样品后, 保持相同速度降温观察液晶相 结构; 傅里叶变换红外光谱仪(FTIR, Bio-Rad FTS-185), 样品用 $\mathrm{CH}_{2} \mathrm{Cl}_{2}$ 溶解后涂覆 $\mathrm{KBr}$ 晶片上, 在不同温度下 扫描 32 次. 紫外-可见分光光度计(UV, Hitachi U2900, 日本); 小角 $\mathrm{X}$ 射线散射 (SAXS, 上海同步光源 BL16B1-X 射线小角散射光束线站, $10 \mathrm{keV}, 1.24 \AA$ ), 液 晶大分子通过熔融拉伸方法制备得到纤维样品; 激光粒 度仪(DLS, ZetaNano ZS90, 英国); 扫描电子显微镜 (SEM, JEOL JSM 6390LV, 日本), $10 \mathrm{kV}$ 加速电压.

\section{3 单体和 RAFT 链转移剂的合成}

甲基丙烯酸丙酮缩甘油酯(SMA)合成 ${ }^{[28]}$ : $50 \mathrm{~mL}$ 含 有三乙胺 $(25.3 \mathrm{~g}, 0.25 \mathrm{~mol})$ 的 $\mathrm{CH}_{2} \mathrm{Cl}_{2}$ 溶液中加入丙酮缩 甘油(33.6 g, $0.25 \mathrm{~mol})$, 冰浴条件下滴加 $30 \mathrm{~mL}$ 含有甲 基丙烯酰氯( $\left(21.8 \mathrm{~g}, 0.21 \mathrm{~mol}\right.$ )的 $\mathrm{CH}_{2} \mathrm{Cl}_{2}$ 溶液, 反应 $2 \mathrm{~h}$ 后 升至室温继续反应 $10 \mathrm{~h}$. 过滤除去不溶物, 水洗两次后, 用无水硫酸钠干燥过夜, 加入 $0.5 \mathrm{~g}$ 阻聚剂亚甲基蓝浓 缩, 然后减压蒸馏 $\left(70{ }^{\circ} \mathrm{C}\right)$ 得产物 $(29 \mathrm{~g}, 0.14 \mathrm{~mol})$, 产率 $69 \%$.

含胆固醇液晶元单体(MA6-Chol)的合成：以胆固 醇为原料, 在室温下将其与对甲苯磺酰氯反应, 丙酮中 重结晶纯化后得到胆固醇对甲苯磺酸酯(Chol-OTs). 接 着在过量己二醇的存在条件下, 将 Chol-OTs 在二氧六
环中加热回流 $12 \mathrm{~h}$ ，二醇中的单个羟基与 OTs 基团置换 成醚，通过柱层析分离纯化后得到 Chol- $\mathrm{O}\left(\mathrm{CH}_{2}\right)_{6} \mathrm{OH}$. 然 后将上述中间产物与甲基丙烯酸在 DCC 和 DMAP 存在 下反应成酯，最终制备得到含有胆固醇液晶元的甲基丙 烯酸酯单体 MA6Chol.

参考文献 ${ }^{[29]}$ 方法合成制备了二硫代苯甲酸 4-氰基 戊酸 $(\mathrm{CPADB})$ 作为 RAFT 链转移剂.

\section{4 液晶嵌段大分子 PGMA-b-PMA6Chol 的合成}

PSMA 的合成: 向预置了搅拌子的干燥 Schlenk 反 应管中加入单体 SMA (1.4 g, $7 \mathrm{mmol})$ 及 $10.5 \mathrm{~mL}$ 甲苯溶 剂, 随后加入链转移剂 CPADB (0.177 g, $0.636 \mathrm{mmol})$ 及 AIBN (0.035 g, $0.212 \mathrm{mmol})$. 将体系冷冻、抽真空、解 冻反复三次, 通氮气循环, 除去反应管中残留氧气，置 于事先预热到 $80{ }^{\circ} \mathrm{C}$ 的恒温油浴中反应 $8 \mathrm{~h}$. 然后液氮冷 冻反应体系终止反应, 将反应液滴加到正己烷溶剂中沉 淀, 真空干燥后得到 $0.98 \mathrm{~g}$ 粉红色固体聚甲基丙烯酸丙 酮缩甘油酯(PSMA), 收率 70\%.

PGMA- $b$-PMA6Chol 的合成: 应用上述反应得到的 PSMA 大分子起始剂引发单体的嵌段共聚. 将三个预置 摚拌子的干燥 Schlenk 反应管编号, 分别按设计好的配 比加入 PSMA, AIBN, MA6Chol 单体及新蒸的甲苯溶剂. 接着将体系经三次冷冻、抽真空、解冻通氮气循环除去 反应管中残留氧气，然后将反应管移入事先预热到 80 ${ }^{\circ} \mathrm{C}$ 的恒温油浴反应 $10 \mathrm{~h}$. 然后将反应液滴加到甲醇溶剂 中沉淀, 并通过三次重复溶解一沉淀对产物进行纯化, 真空干燥后得到粉红色固体状 PSMA- $b$-PMA6Chol. 在 此基础上，称取 $200 \mathrm{mg}$ 干燥后的 PSMA- $b$-PMA6Chol 样品溶于 $10 \mathrm{~mL}$ 的 $\mathrm{THF}$ 中, 搅拌条件下慢慢滴加 1.0 $\mathrm{mol} / \mathrm{L} \mathrm{HCl}$ 溶液 $1.5 \mathrm{~mL}$, 室温下反应 $24 \mathrm{~h}$. 反应结束后 将反应液浓缩并滴入甲醇-正已烷(2：1)溶剂中沉淀， 重复两次溶解一沉淀, 过滤分离真空干燥后得到粉红色 目标产物 PGMA- $b$-PMA6Chol.

\section{5 溶液自组装}

将制备所得的两亲性嵌段液晶大分子溶于两嵌段 的共溶剂 THF 中, 初始浓度为 $0.1 \mathrm{wt} \%$. 充分溶解 $12 \mathrm{~h}$ 后，缓慢向溶液中滴加去离子水并轻微摇动以混合均 匀, 加水速度为 $2.0 \mu \mathrm{L} / \mathrm{min}$, 每次加水后溶液静置 $5 \mathrm{~min}$ 使其达到平衡, 然后通过紫外-可见分光光度计(波长 $650 \mathrm{~nm})$ 对加水后的混合溶液的透光率 $(T \%)$ 检测, 混合 溶液的水含量控制在 $60 \mathrm{wt} \%$ 之内. 最终得到的混合溶 液使用截留分子量为 $3500 \mathrm{Da}$ 的再生纤维素渗透膜透 析, 每 $8 \mathrm{~h}$ 更换一次去离子水, 透析 3 天, 直至有机溶剂 完全去除, 进一步采用 DLS 和 SEM 对自组装聚集体的 粒径和形貌结构进行研究. 不同温度条件下考察自组装 聚集体的粒径变化是将室温自组装后的透析液加入无 尘石英血中, 使用 DLS 仪器配置的恒温槽在每个温度 点上恒温 $45 \mathrm{~min}$ 使聚集体变化达到平衡, 然后重复测量 
粒径三次取平均值. 同时采用 SEM 分别在 $60{ }^{\circ} \mathrm{C}$ 和升 温-降温循环后取样制得的样品进行形貌表征，其中 60 ${ }^{\circ} \mathrm{C}$ 条件的样品操作如下: 待溶液于 $60{ }^{\circ} \mathrm{C}$ 下充分恒温后, 取样滴于同样也在 $60{ }^{\circ} \mathrm{C}$ 下恒温后的云母片上, 然后在 $60{ }^{\circ} \mathrm{C}$ 的环境下干燥, 以固定 $60{ }^{\circ} \mathrm{C}$ 条件下聚集体的形 貌.

\section{致谢}

本文作者对相关前期研究工作中得到法国国家研 究中心-中国科学院(CNRS-CAS)中法国际合作项目的 支持表示感谢, 同时感谢法国居里研究所物理化学实验 室李敏慧研究员对本文研究工作的帮助.

\section{References}

[1] Sun, T.-M.; Du, J.-Z.; Yan, L.-F.; Mao, H.-Q.; Wang, J. Biomaterials 2008, 29, 4348 .

[2] Rouzes, C.; Durand, A.; Leonard, M.; Dellacherie, E. J. Colloid Interface Sci. 2002, 253, 217

[3] Zhu, Y.-M.; Zhang, Y.; Liu, Z.-L.; Lang, M.-D. Acta Chim. Sinica 2010, 68, 2449. (朱亚明, 张琰, 刘子路, 郎美东, 化学学报, 2010, $68,2449$.

[4] Chen, X.-F.; Shen, Z.-H.; Wan, X.-H.; Fan, X.-H.; Chen, E.-Q.; Ma, Y.-G.; Zhou, Q.-F. Chem. Soc. Rev. 2010, 39, 3072.

[5] Liang, X.-C.; Chen, X.-F.; Li, C.-Y.; Shen, Z.-H.; Fan, X.-H.; Zhou, Q.-F. Acta Polym. Sin. 2011, (11), 1311. (梁小朝, 陈小芳, Li Christopher Y., 沈志豪，范星河，周其风，高分子学报， 2011, (11), 1311.)

[6] Tschierske, C. Chem. Soc. Rev. 2007, 36, 1930.

[7] Mabrouk, E.; Cuvelier, D.; Brochard-Wyart, F.; Nassoy, P.; Li, M.-H. Proc. Natl. Acad. Sci. U. S. A. 2009, 106, 7294.
[8] Jia, L.; Daniel, L.; Dominique, D.; Marianne, I.-C.; Cao, A.-M.; Li, M.-H. Soft Matter 2011, 7, 7395.

[9] Tang, J.-J.; Li, J.-G.; Qi, W.; Qiu, W.-W.; Li, P.-S.; Li, B.-L.; Song, B.-L. Cell Metabolism 2011, 13, 44

[10] Sheng, R.-L.; Luo, T.; Zhu, Y.-D.; Li, H.; Sun, J.-J.; Chen, S.-D.; Sun, W.-Y.; Cao, A.-M. Biomaterials 2011, 32, 3507.

[11] Pinol, R.; Jia, L.; Gubellini, F.; Levy, D.; Albouy, P.-A.; Keller, P.; Cao, A.-M.; Li, M.-H. Macromolecules 2007, 40, 5625

[12] Jia, L.; Cao, A.-M.; Levy, D.; Xu, B.; Albouy, P.-A.; Xing, X.; Bowick, M.; Li, M.-H. Soft Matter 2009, 5, 3446.

[13] Chen, S.-D.; Hu, F.-Z.; Sheng, R.-L.; Cao, A.-M. Acta Polym. Sin. 2013，(1)，102. (陈圣典，胡方振，盛瑞隆，曹阿民，高分子学报， 2013, (1), 102.)

[14] Gates, G.; Harmon, J.-P.; Ors, J.; Benz, P. Polymer 2003, 44, 207.

[15] Gates, G.; Harmon, J.-P.; Ors, J.; Benz, P. Polymer 2003, 44, 215.

[16] Leung, B.-K.; Robinson, G.-B. J. Appl. Polym. Sci. 1993, 47, 1207.

[17] Duong, H.-T. T.; Marquis, C.-P.; Whittaker, M.; Davis, T.-P.; Boyer, C. Macromolecules 2011, 44, 8008.

[18] Wang, S.-Y.; Pelet, J.-M.; Putnam, D. Prog. Polym. Sci. 2007, 32, 799.

[19] Zhou, Y.; Ahn, S.; Lakhman, R.-K.; Gopinadhan, M.; Osuji, C.-O.; Kasi, R. M. Macromolecules 2011, 44, 3924.

[20] Baljinder, K.; Larissa, D.; Thomas, H.-M.; Liang, S.-W.; Warren, T.; Ford, W.-T. Macromolecules 2011, 44, 3810.

[21] Yang, J.; Zhang, D.-W.; Jiang, S.; Yang, J.-J.; Nie, J. J. Colloid Interface Sci. 2010, 352, 405.

[22] Kyeremateng, S.-O.; Amado, E.; Kressler, J. Eur. Polym. J. 2007, $43,3387$.

[23] Zhang, X.-W.; Boisse, S.; Bui, C.; Albouy, P.-A.; Brulet, A.; Li, M.-H.; Rieger, J.; Charleux, B. Soft Matter 2012, 8, 1130.

[24] Yu, K.; Zhang, L.; Eisenberg, A. Langmuir 1996, 12, 5980.

[25] Ga, Z.-S.; Varshney, S.-K.; Eisenberg, A. Macromolecules 1994, 27, 7923.

[26] Xu, J.-T.; Tao, L.; Boyer, C.; Lowe, A.-B.; Davis, T.-P. Macromolecules 2011, 44, 299.

[27] Kyeremateng, S.-O.; Henze, T.; Busse, K.; Kressler, J. Macromolecules 2010, 43, 2502.

[28] Kibret, M.; Alpesh, P.; Deon, B. Biomacromolecules 2006, 7, 883.

[29] Zheng, Q.; Pan, C.-Y. Macromolecules 2005, 38, 6841.

(Cheng, B.) 